\title{
Interaction between favipiravir and hydroxychloroquine and their combined drug assessment: in silico investigations
}

\author{
Cemal Parlak ${ }^{1}$. Özgür Alver ${ }^{2} \cdot$ Cecil Naphtaly Moro Ouma ${ }^{3} \cdot$ Lydia Rhyman $^{4,5} \cdot$ Ponnadurai Ramasami $^{4,5}$
}

Received: 19 May 2021 / Accepted: 22 October 2021 / Published online: 2 November 2021

(c) Institute of Chemistry, Slovak Academy of Sciences 2021

\begin{abstract}
Hydroxychloroquine (HCQ) and favipiravir (FPV) are known to be effective antivirals, and there are reports about their use to fight the severe acute respiratory syndrome coronavirus 2 (SARS-CoV-2) despite that these are not conclusive. The use of combined drugs is common in drug discovery, and thus, we investigated HCQ and FPV as a combined drug. The density functional theory method was used for the optimization of geometries, spectroscopic analysis and calculation of reactivity parameters. The quantum theory of atoms in molecules was applied to explain the nature of the hydrogen bonds and confirm the higher stability of the combined drug. We also evaluated the absorption, distribution, metabolism and excretion (ADME) parameters to assess their drug actions jointly using SwissADME. The preliminary findings of our theoretical study are promising for further investigations of more potent and selective antiviral drugs.
\end{abstract}

\section{Graphical abstract}

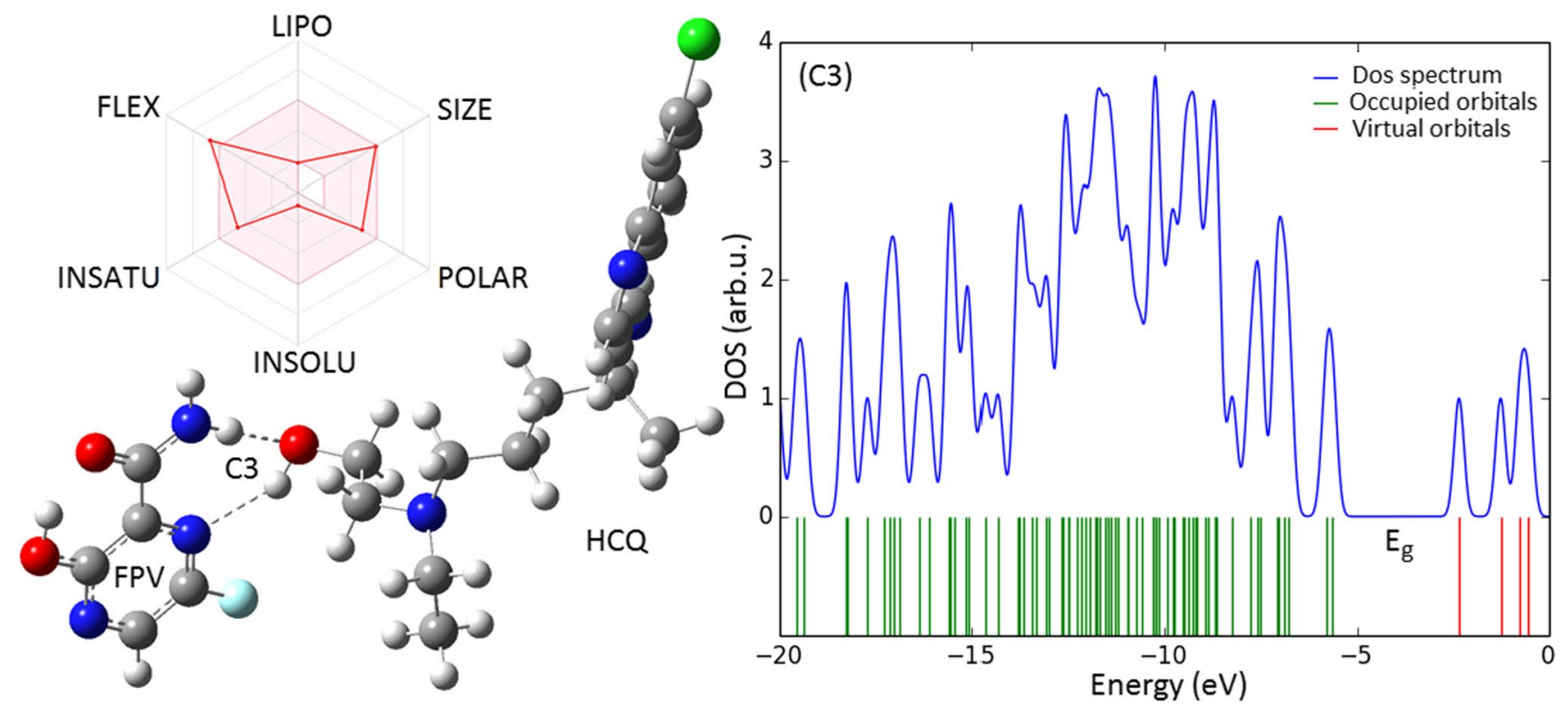

Keywords Favipiravir $\cdot$ Hydroxychloroquine $\cdot$ Drug interactions $\cdot$ DFT $\cdot$ QTAIM $\cdot$ ADME

Ponnadurai Ramasami

p.ramasami@uom.ac.mu

Extended author information available on the last page of the article

\section{Introduction}

In late 2019, a new type of coronavirus named SARS-CoV-2 appeared in Wuhan, China, and rapidly spread all over the World (Lai et al. 2020). To date, there have already been 


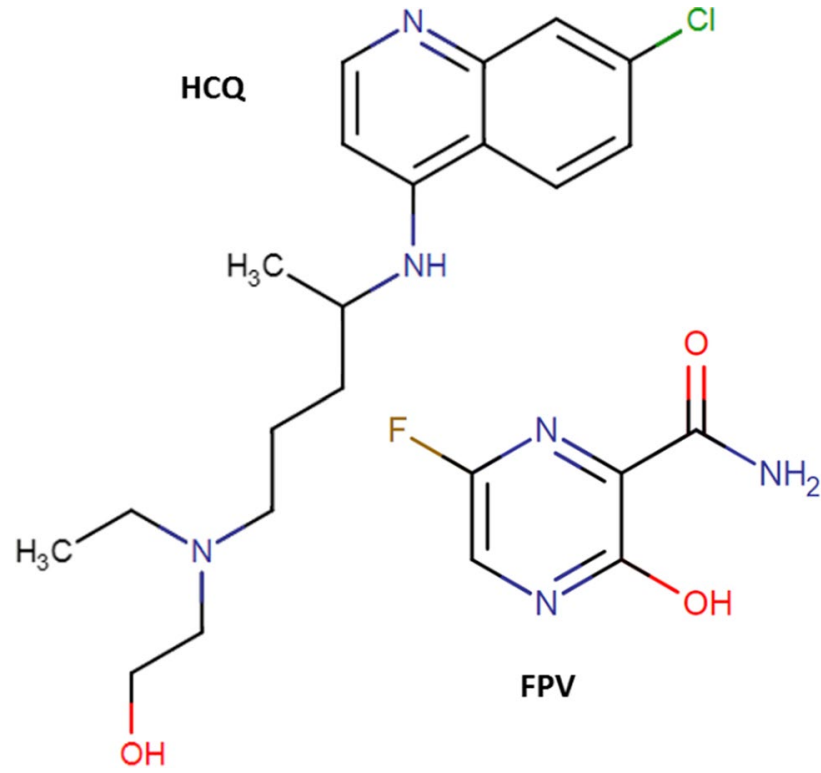

Scheme 1 Molecular structure of HCQ and FPV

$164,929,440$ persons being infected worldwide and about 3,419,966 persons lost their lives (COVID-19: Coronavirus Pandemic, Last updated 2021). One of the ways to combat the spread of this highly contagious virus has been to adopt confinement (Zhang et al. 2020), and this has led to huge economic lost (McKibbin and Fernando 2020) among several issues (Wong et al. 2020). Thus, there is an urgent need to identify suitable and reliable drugs (Le et al. 2020). One of the different strategies is to rely on existing drugs as the safety profiles of these drugs are already established (Colson et al. 2019; Gautret et al. 2020). Another strategy is to use combinations of drugs so as to improve the pharmacodynamics and pharmacokinetic efficacy (Flynn 2007). Although vaccination appears to be the most effective way to prevent an outbreak for a particular infectious disease, the development and the assessment of a vaccine require a lot of time. Nevertheless, there are many vaccines are available that have proven to be successful such as influenza, HPV and chickenpox vaccines (Fiore et al. 2009; Chang et al. 2009; Liesegang 2009).

FPV also known as T-705, as shown in Scheme 1, is an antiviral agent, and it is also known for its broad spectral activity against ribonucleic acid (RNA) viruses (Furuta et al. 2009). This drug was approved by Shenzan Health Commission for the possible treatment of COVID-19 patients (Wu et al. 2020). HCQ is another drug (Scheme 1) which has been reported for the possible treatment of COVID-19 patients (Gautret et al. 2020; Singh et al. 2020a). There are reports indicating that FPV and HCQ have some positive effects on the treatment of less severe COVID-19 patients (Singh et al. 2020b).
QTAIM analysis has been a useful tool for the examination of intra- and intermolecular hydrogen bonding (HB) interactions (Bader 1990; Savin et al. 1997) although several objections were raised about the terminology preferred (Shahbazian 2018; Jabłoński 2019). At the base of this theory, three parameters of bond critical points (BCP), electron density $\rho(r)$, Laplacian of electron density $\nabla^{2} \rho(r)$ and electronic energy density $\mathrm{H}(\mathrm{r})$ are important to assess the nature of interaction (Bader 1990). According to Rozas et al. (Rozas et al. 2000), the nature of HB is defined as strong if $\nabla^{2} \rho(r)$ and $H(r)$ are negative. If $\nabla^{2} \rho(r)$ is positive and $H(r)$ is negative, then the HB interaction is intermediate or partially covalent. Finally, if $\nabla^{2} \rho(r)$ and $H(r)$ are positive, then the HB interactions are called as weak or non-covalent (Rozas et al. 2000).

The ability of electron donation and electron acceptance for a given molecular system can be assessed by analyzing highest occupied molecular orbital (HOMO) and lowest unoccupied molecular orbital (LUMO). The HOMO-LUMO energy gap is a well-known parameter to evaluate molecular charge transfers processes. They are also used for the identification of chemical reactivity, bioactivity, the stability and general reactivity of molecular systems (Fukui 1982; Parr et al. 1999; Samsonowicz et al. 2017).

Density functional theory (DFT) method has been widely applied for the examination of the structural, electronic and spectroscopic properties of different compounds (Hao et al. 2019; Astani et al. 2016). DFT is a useful tool for modeling drug design and the examination of molecular interactions since it is cost-effective and faster than the time-consuming experimental methods (Astani et al. 2016; Pierens et al. 2017; Khorram et al. 2017). In addition to ab initio method, the SwissADME web tool (Daina et al. 2017) is commonly used to have drug-related parameters.

In view of the above resume and in continuation with our interests to study drugs using theoretical methods (Rhyman et al. 2018; Alver et al. 2019; Parlak and Alver 2017), we were interested to investigate FPV and HCQ working jointly as a drug. Within this aim, we set objectives, namely: (i) to use DFT and QTAIM to understand the nature of the interactions between the two drugs and (ii) to use SwissADME to have insights in the drug capabilities of the combined drugs. We hereby report the findings of our research.

\section{Computational details}

All ab initio calculations were performed using Gaussian 09 (Frisch et al. 2009) within the DFT framework with the B3LYP functional in conjunction with the 6-31G(d) basis set for all atoms. The polarizable continuum model was used to include the solvation effect (Tomasi et al. 2005). Molecular structures and initial geometrical 
configurations were built and visualized with the GaussView program (Dennington et al. 2009). Firstly, isolated FPV and HCQ were optimized in water without imposing any structural limitations. Geometry optimizations were followed by vibrational frequency calculations to ensure that the resultant structures converge to a ground state or a minimum energy state. An analysis of the lowest energy configurations natural bond orbital (NBO) charge (Reed et al. 1988) was conducted to identify the possible interaction edges between FPV and HCQ. After having decided the possible interaction edges for FPV and HCQ, the combined drugs were optimized until no imaginary frequency was obtained.

We followed two steps to determine the most possible geometry of the interacted drugs. Initially, binding energies $\left(\mathrm{E}_{\mathrm{b}}\right)$ of the possible drug couples are calculated using Eq. (1) (Zhai et al. 2019):

$\mathrm{E}_{\mathrm{b}}=\mathrm{E}_{\text {combined }}-\left[\mathrm{E}_{\mathrm{FPV}}+\mathrm{E}_{\mathrm{HCQ}}\right]$

In Eq. (1), $\mathrm{E}_{\text {combined }}, \mathrm{E}_{\mathrm{FPV}}$ and $\mathrm{E}_{\mathrm{HCQ}}$ are the optimized electronic energies of the investigated systems. It is known that a negative value of $E_{b}$ reflects a favorable process. Consequently, a positive value of $\mathrm{E}_{\mathrm{b}}$ means an unfavorable process. After the optimizations, the structures with positive $\mathrm{E}_{\mathrm{b}}$ values were not considered for further investigations. It is also known that at the sites where the interactions take place, basis functions overlap and this leads to an error known as basis set superposition error (BSSE) causing some alterations in the $\mathrm{E}_{\mathrm{b}}$ energies of the investigated molecular systems (Simon et al. 1996; Zheng et al. 2016). Thus, in the present study, the BSSE errors were included by using the counterpoise correction method (Boys and Bernardi 1970). After eliminating the unlikely candidates of drug couples, a topological analysis of electron density was carried out with QTAIM analyses for the structures having negative $E_{b}$ values. QTAIM calculations were performed by the Multiwfn program (Lu and Chen 2012). SwissADME web tool was used to predict the drug-related parameters (SwissADME: a free web tool to evaluate pharmacokinetics, drug-likeness and medicinal chemistry friendliness of small molecules. 2017).

\section{Results and discussion}

The coordinates of the optimized structures and the dipole moments FPV, HCQ and interacted drugs (C1-C5) are collected in Tables SI1-7 (SI: Supplementary information). The optimized structures (five possibilities) of the interacted drugs are displayed in Fig. 1 with the $\mathrm{E}_{\mathrm{b}}$ energy. The calculated $\mathrm{E}_{\mathrm{b}}$ values are found to be positive for $\mathrm{C} 1, \mathrm{C} 4$ and $\mathrm{C} 5$ and thus unfavorable. Therefore, in this section, the
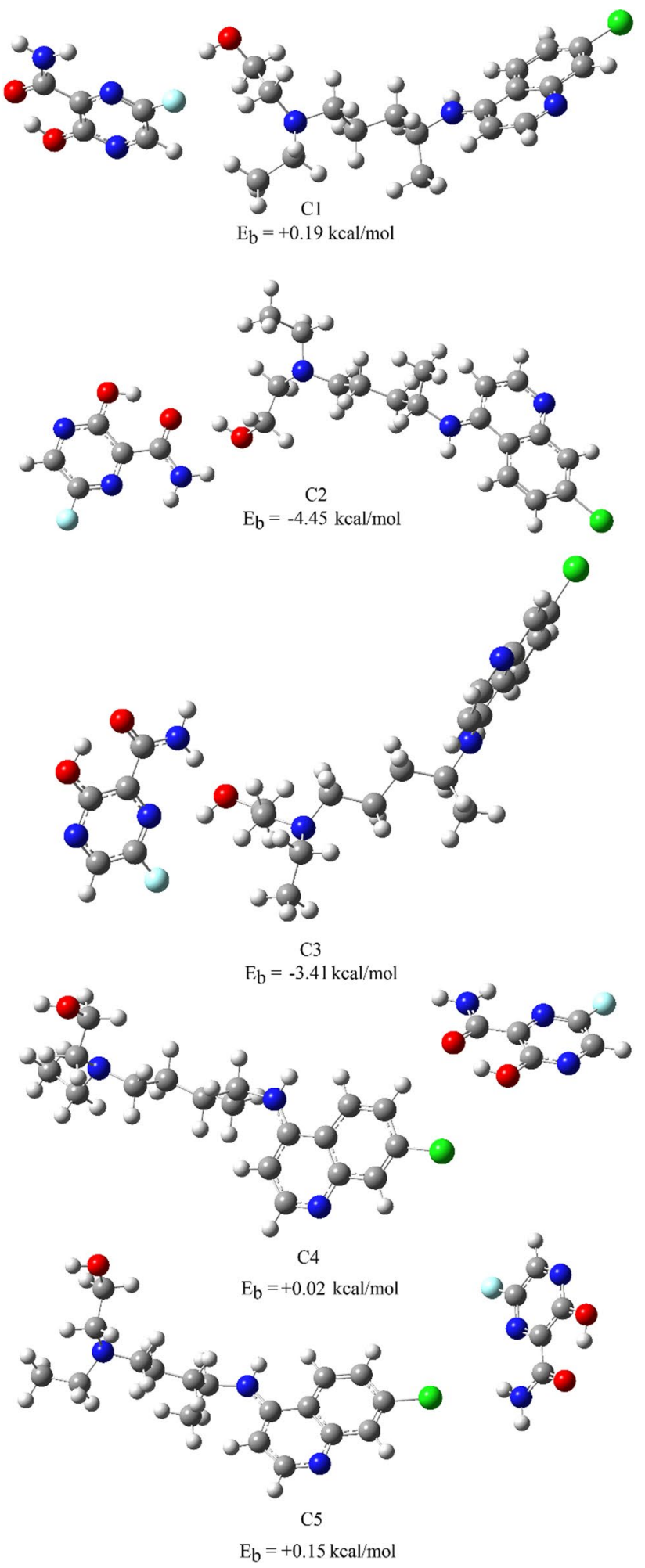

Fig. 1 Optimized structures with $\mathrm{E}_{\mathrm{b}}$ energies

discussion is limited to $\mathrm{C} 2$ and $\mathrm{C} 3$ structures since they have exothermic binding energies values of $-4.45 \mathrm{kcal} / \mathrm{mol}$ and $-3.41 \mathrm{kcal} / \mathrm{mol}$. 


\section{Analysis of C2 interaction}

The molecular topography map and some important topological parameters of $\mathrm{C} 2$ are given in Fig. 2. In this representation, BCPs are shown by orange spheres. It can be observed from Fig. 2 that QTAIM calculations provide two possible intermolecular HB interactions, namely site I and site II. At the site I, interaction occurs via $\mathrm{O}-\mathrm{H}$... O group. For this edge, the $\nabla^{2} \rho(r)$ was calculated as positive and the value of $\mathrm{H}(\mathrm{r})$ was found to be negative, and thus, these indicate the partial covalent interaction with $\mathrm{E}_{\mathrm{HB}}$ value of $-8.02 \mathrm{kcal} /$ mol. At site II, interaction occurs via $\mathrm{N}-\mathrm{H}$...O groups with $\mathrm{E}_{\mathrm{HB}}$ value of $-6.37 \mathrm{kcal} / \mathrm{mol}$. It is seen that the $\mathrm{HB}$ character at site I is stronger than site II leading to a larger electron density at BCP.

In $\mathrm{C} 2$, the effect of interaction can be best followed from the hydroxyl edge of HCQ drug since it is easy to identify the characteristic $\mathrm{OH}$ vibration by the infrared (IR) spectroscopic method. For a single isolated HCQ, the OH stretching vibration is observed at $3737 \mathrm{~cm}^{-1}$ with an IR intensity of $26.8 \mathrm{~km} / \mathrm{mol}$ (Fig. 3). After interaction with FPV, this band shifts down to $3541 \mathrm{~cm}^{-1}$ and IR intensity increases to $747.2 \mathrm{~km} / \mathrm{mol}$. This red-shift indicates a weakening in the bond character of $\mathrm{OH}$. As a result, the force constant of $\mathrm{OH}$ bond of HCQ decreases from 8.77 to $7.89 \mathrm{mdyn} / \AA$ upon interaction with FPV. The reduction in the force constant causes a change in the $\mathrm{OH}$ bond length of HCQ from $0.971 \AA$ to $0.982 \AA$ after interaction with FPV (Fig. 3).

HOMO-LUMO distributions over the surface of a given molecular structure provide useful information for the identification of molecular activity. For example, the more negative LUMO energy values are considered as the descriptors of chemically more active molecules (Fukui 1982; Ayala and Scuseria 1999; Kawakami et al. 2013). It was previously reported that molecular compounds with more stabilized LUMO orbitals have higher biological activities (Kumar et al. 2018). It was also reported that the molecular sites

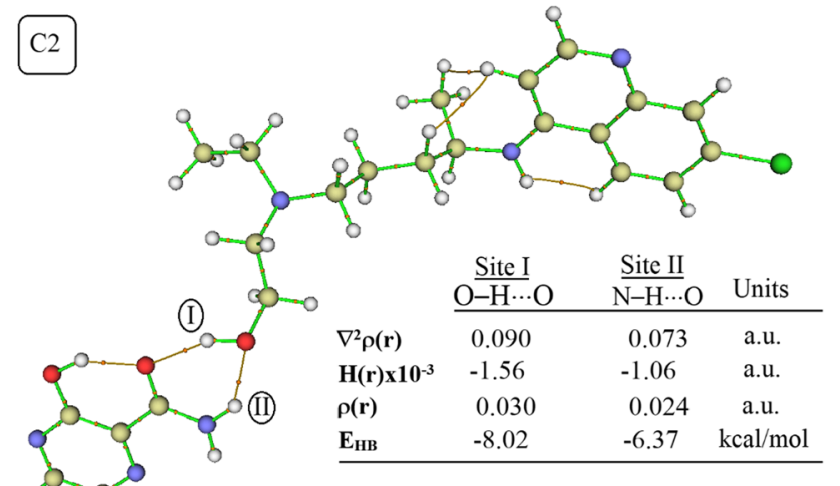

Fig. 2 Molecular topography map of C2

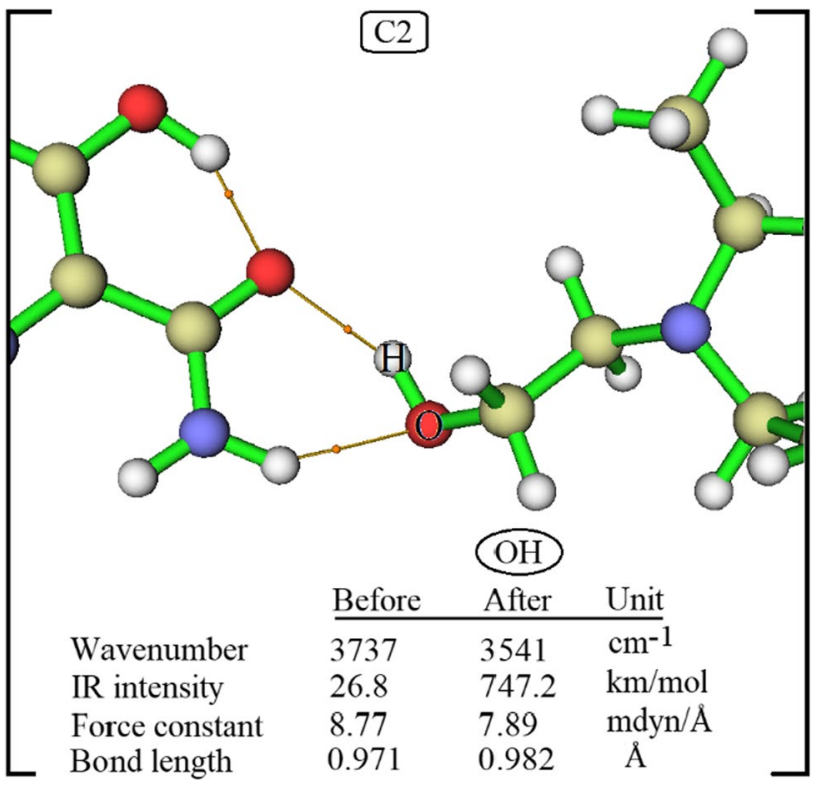

Fig. 3 Vibrational properties and bond characteristics at the interaction site of $\mathrm{C} 2$

where the LUMO orbitals are distributed play an important role for the biological activities (Kawakami et al. 2013; Kumar et al. 2018). Furthermore, ionization potential (I) estimated as $-\mathrm{E}_{\mathrm{HOMO}}$ and electron affinity (A) estimated as $-\mathrm{E}_{\text {LUMO }}$ are also considered as general reactivity identifiers (Samsonowicz et al. 2018). HOMO-LUMO plots and calculated energy values for $\mathrm{C} 2$ are given in Fig. 4 . The HOMO orbital is located on HCQ, and the LUMO orbital is distributed over FPV. These results indicate that while HCQ behaves as an electron donor, FPV behaves as an electron acceptor. $\mathrm{E}_{\mathrm{LUMO}}$ values of single isolated FPV and HCQ were calculated as $-2.27 \mathrm{eV}$ and $-1.26 \mathrm{eV}$. After the interaction, $\mathrm{E}_{\mathrm{LUMO}}$ of $\mathrm{C} 2$ becomes more negative with a value of $-2.33 \mathrm{eV}$ suggesting an increase in the chemical activity and the electron affinity of the $\mathrm{C} 2$ system compared to

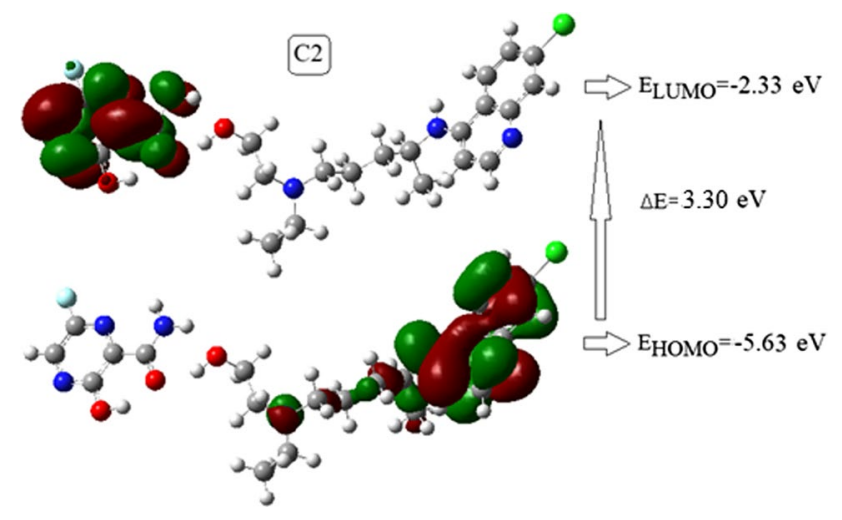

Fig. 4 Frontier molecular orbitals of $\mathrm{C} 2$ 
FPV and HCQ separately. There is also an increase in the activity of FPV for the interacted C2 system since LUMO is located over the FPV following the interaction. $\mathrm{E}_{\mathrm{g}}$ value of $\mathrm{C} 2(3.30 \mathrm{eV})$ is found to be smaller than FPV $(4.57 \mathrm{eV})$ and HCQ $(4.37 \mathrm{eV})$, and this also shows that the chemical reactivity of $\mathrm{C} 2$ is higher than FPV and HCQ alone.

\section{Analysis of C3 interaction}

The molecular topography map along with some important topological parameters of $\mathrm{C} 3$ is provided in Fig. 5. The QTAIM calculations for $\mathrm{C} 3$ indicate two possible intermolecular HB interactions labeled as site I and site II. At site I, interaction occurs via $\mathrm{N}-\mathrm{H}$...O groups. For this edge, it is found that $\nabla^{2} \rho(\mathrm{r})>0$ and $\mathrm{H}(\mathrm{r})<0$ and these refer to a partial covalent interaction with $\mathrm{E}_{\mathrm{HB}}$ value of $-8.04 \mathrm{kcal} / \mathrm{mol}$. At site II $(\mathrm{O}-\mathrm{H} \ldots \mathrm{N})$, both $\nabla^{2} \rho(\mathrm{r})$ and $\mathrm{H}(\mathrm{r})$ are found to be positive indicating a non-covalent interaction with $\mathrm{E}_{\mathrm{HB}}$ value of $-4.92 \mathrm{kcal} / \mathrm{mol}$. Electron density at $\mathrm{BCP}$ was found larger for site I interaction (Fig. 5).

In $\mathrm{C} 3$, the $\mathrm{OH}$ stretching vibration of HCQ is red shifted to $3658 \mathrm{~cm}^{-1}$ as a result of $\mathrm{HB}$ interaction and IR intensity increases to $348 \mathrm{~km} / \mathrm{mol}$ (Fig. 6). The force constant of $\mathrm{OH}$ bond of HCQ decreases to $8.43 \mathrm{mdyn} / \AA$ upon interaction with FPV. Consequently, the weakening of the force constant causes an increase in the OH bond length of HCQ from 0.971 to $0.976 \AA$ following the interaction with PFV. The HOMO orbital is located on HCQ and the LUMO orbital is in FPV. The $\mathrm{E}_{\mathrm{LUMO}}$ value of $\mathrm{C} 3$ is found to be more negative $(-2.34 \mathrm{eV})$ compared to FPV $(-2.27 \mathrm{eV})$ and HCQ $(-1.26 \mathrm{eV})$ separately. As shown from Fig. 7, $\mathrm{E}_{\mathrm{g}}$ value of $\mathrm{C} 3(3.29 \mathrm{eV})$ is also found to be smaller than drugs $(4.57 \mathrm{eV}$ and $4.37 \mathrm{eV})$. $E_{g}$ and $E_{\text {LUMO }}$ values are found to be slightly smaller than C2. These indicate an increase in activity following the interaction.

C3

\begin{tabular}{|c|c|c|c|}
\hline & $\frac{\text { Site I }}{N-\mathrm{H} \cdots \mathrm{O}}$ & $\frac{\text { Site II }}{\mathrm{O}-\mathrm{H} \cdots \mathrm{N}}$ & Units \\
\hline$\nabla^{2} \rho(\mathbf{r})$ & 0.087 & 0.064 & a.u. \\
\hline $\mathrm{H}(\mathrm{r}) \times 10^{-3}$ & -1.90 & 0.15 & a.u. \\
\hline$\rho(\mathbf{r})$ & 0.030 & 0.021 & a.u. \\
\hline $\mathbf{E}_{\mathrm{HB}}$ & -8.04 & -4.92 & $\mathrm{kcal} / \mathrm{mol}$ \\
\hline
\end{tabular}

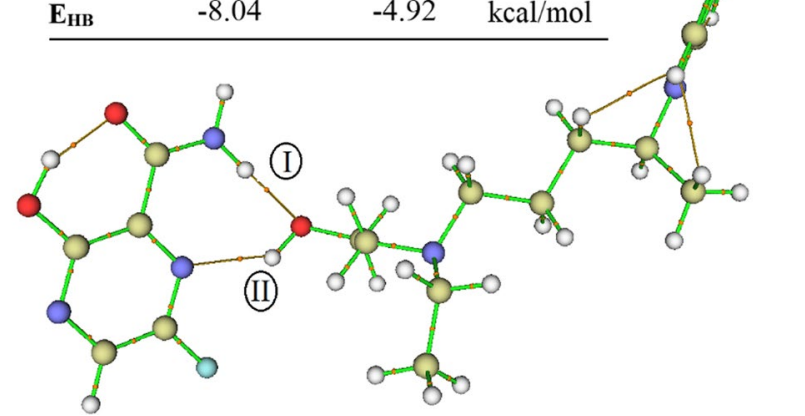

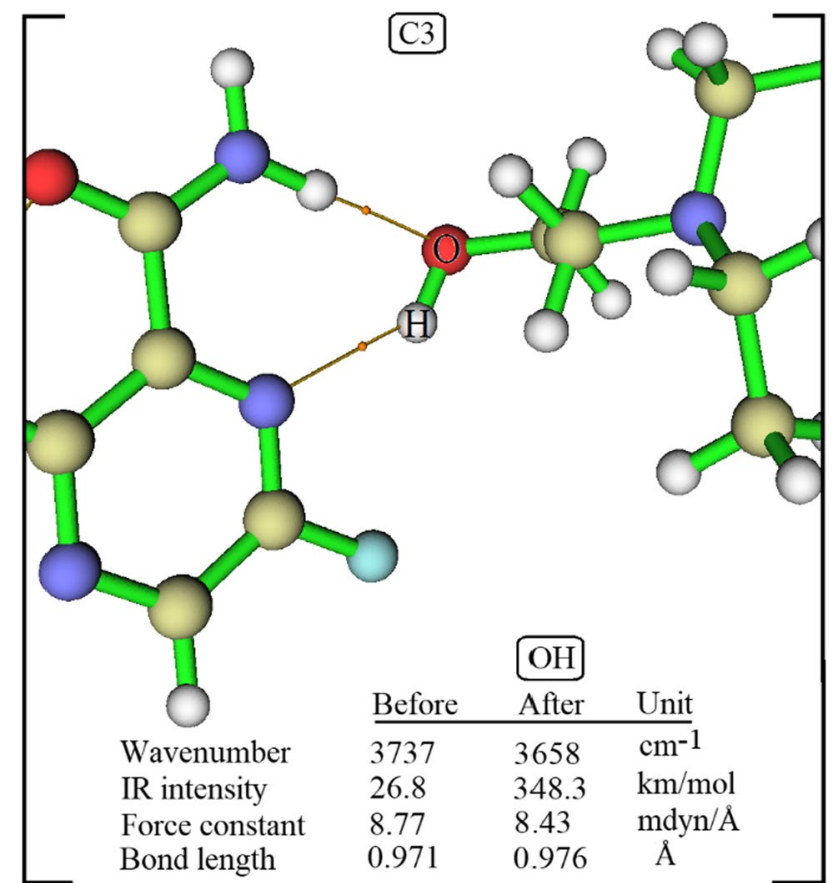

Fig. 6 Vibrational properties and bond characteristics at the interaction site of $\mathrm{C} 3$

\section{SwissADME analysis}

We predicted the physicochemical properties and drug-likeness of FPV, HCQ and their combined structure. Physicochemical properties give a global description of the structure of the compound including molecular weight $(\mathrm{g} / \mathrm{mol})$, molecular refractivity, topological polar surface area $\left(\AA^{2}\right)$,

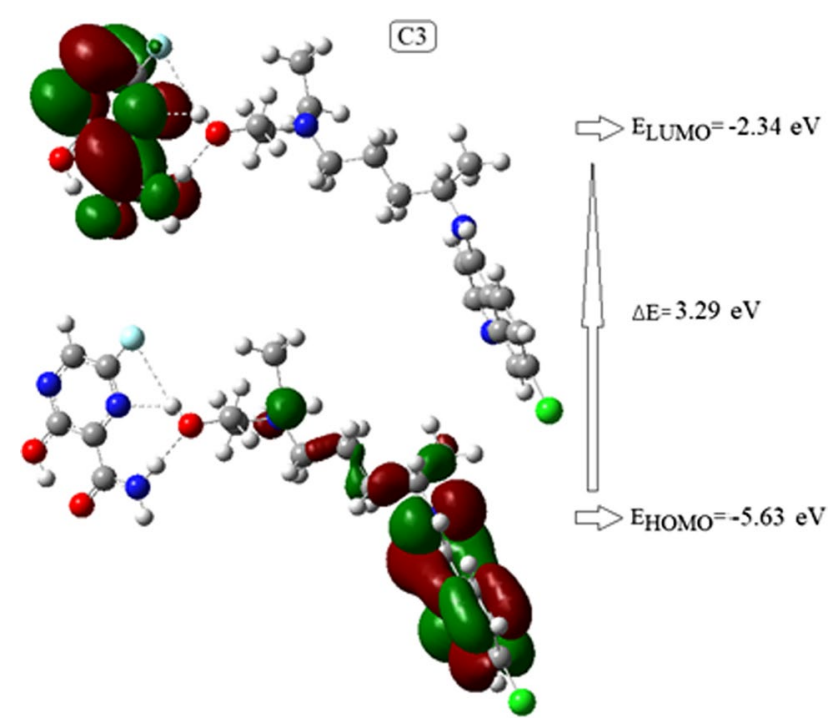

Fig. 7 Frontier molecular orbitals of C3

Fig. 5 Molecular topography map of C3 
number of rotatable bonds, heavy atoms and hydrogen bond acceptors and donors (Table 1).

The bioavailability radar of the compounds displayed a rapid evaluation of drug-likeness. It includes the following six physicochemical properties: (1) Lipophilicity (XLOGP3 between -0.7 and +5.0 ), (2) size (molecular weight between 150 and $500 \mathrm{~g} / \mathrm{mol}$ ), (3) polarity (the total polar surface area between 20 and $130 \AA^{2}$ ), (4) solubility (log S not higher than 6), (5) saturation (fraction Csp3 not less than 0.25) and (6) flexibility (the number of rotatable bonds not more than 9).

The bioavailability radar of FPV, HCQ and upon interaction for six physicochemical properties is shown in Fig. 8. The pink area represents the optimal range of these properties, and the red line represents the properties of the compounds. In Fig. 8, the red lines of the combined compound are in the range of the pink area except for the flexibility value (slightly). Therefore, we can conclude that the combined drugs are predicted orally as bioavailable.

Furthermore, drug-likeness was established based on the physicochemical properties to find oral drug candidates.

Table 1 Physicochemical properties and lipophilicity of FPV, HCQ and FPV-HCQ

\begin{tabular}{llll}
\hline Properties & FPV & HCQ & FPV-HCQ \\
\hline Molecular weight & 157.10 & 335.87 & 492.97 \\
Heavy atom & 11 & 23 & 34 \\
Arom. heavy atom & 0 & 0 & 0 \\
Fraction Csp3 & 0.00 & 0.56 & 0.43 \\
Rotatable bond & 1 & 9 & 10 \\
H-Bond acceptor & 5 & 3 & 8 \\
H-Bond donor & 2 & 2 & 4 \\
Molar refractivity & 45.10 & 102.41 & 147.51 \\
Polar surface area & 63.32 & 35.50 & 98.82 \\
Lipophilicity & & & \\
MLOGP & -1.84 & 1.59 & -0.39 \\
WLOGP & -2.89 & 1.42 & -1.46 \\
XLOGP3 & -2.89 & 0.70 & -2.20 \\
\hline
\end{tabular}

There are five different rule-based filters which are defined as follows:

(1) Lipinski's filter includes molecular weight $\leq 500$, MLOGP (lipophilicity) $\leq 4.15$, hydrogen bond acceptors $\leq 10$ and hydrogen bond donors $\leq 5$.

(2) Ghose's filter includes $160 \leq$ molecular weight $\leq 480,-0.4 \leq$ WLOGP (lipophilicity) $\leq 5.6,40 \leq$ the molar refractivity $\leq 130$ and $20 \leq$ number of atoms $\leq 70$.

(3) Veber's filter includes the number of rotatable bonds $\leq 10$ and the total polar surface area $\leq 140$.

(4) Egan's filter includes WLOGP (Lipophilicity) $\leq 5.88$ and the total polar surface area $\leq 131.6$.

(5) Muegge's filter includes $200 \leq$ molecular weight $\leq 600,-2 \leq$ XLOGP3 (lipophilicity) $\leq 5$, the total polar surface area $\leq 150$, the number of rings $\leq 7$, the number of carbon $>4$, the number of heteroatoms $>1$, the number of rotatable bonds $\leq 15$, the hydrogen bond acceptors $\leq 10$ and the hydrogen bond donors $\leq 5$.

The result of the drug-likeness evaluation of FPV, HCQ and upon interaction is shown in Table 2. The combined drug is in agreement with the Lipinski's, Veber's and Egan's rules. Turning to Ghose's four rules, it is in agreement with one rule excluding MW $>480$, MR $>130$ and WLOGP $<-0.4$. The combined drug passes to Muegge's nine rules excluding one lipophilicity value $(\mathrm{XLOGP} 3<-2)$. It can be concluded that this interaction is predicted orally bioavailable. These preliminary results provide the lead for the design of more potent and selective antiviral drugs.

Table 2 Drug-likeness evaluation of FPV, HCQ and FPV-HCQ

\begin{tabular}{llll}
\hline Rule-based filters & FPV & HCQ & FPV-HCQ \\
\hline Lipinski & Yes & Yes & Yes \\
Ghose & No; 3 violations & Yes & No; 3 violations \\
Veber & Yes & Yes & Yes \\
Egan & Yes & Yes & Yes \\
Muegge & No; 2 violation & Yes & No; 1 violations \\
\hline
\end{tabular}

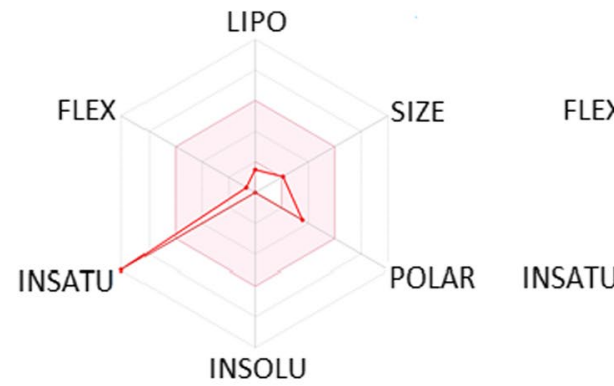

(a)

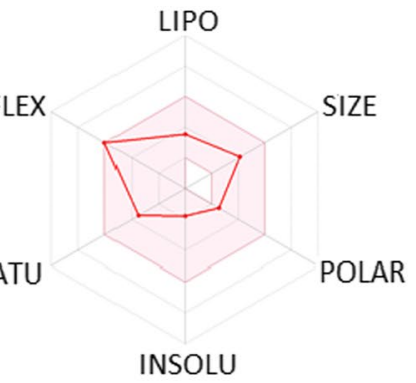

(b)

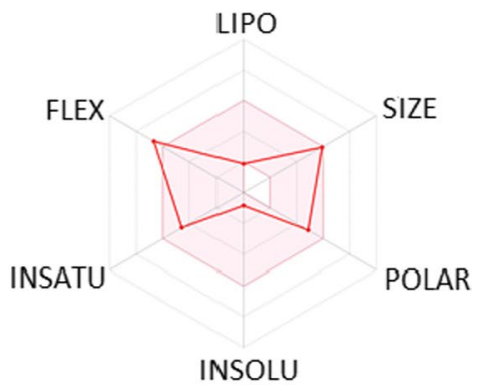

(c)

Fig. 8 Bioavailability radar of FPV (a), HCQ (b) and (c) FPV-HCQ 


\section{Conclusions}

The interaction mechanism, structural, reactivity and spectroscopic examinations of the combined FPV and HCQ drugs were investigated with DFT and QTAIM approaches. The drug-likeness evaluations of FPV-HCQ were also performed by ADME parameters.

It was observed that following the interaction LUMOs were located over the FPV and $\mathrm{E}_{\mathrm{LUMO}}$ values of the interacted systems became more negative indicating an increase in the antiviral activity and electron affinity. The charge transfer was found from HCQ to FPV. It is shown that for the drug interactions occurring through electron acceptance, low LUMO energy values suggest that FPV-HCQ couple shows higher activities via LUMOs distributing over FPV when compared to single FPV. It is also observed that HB interactions with bond energies almost $-8 \mathrm{kcal} / \mathrm{mol}$ lead to a red-shift for the hydroxyl stretching vibration of HCQ. Further, the IR intensity and bond length of the hydroxyl group increase as a result of HB interactions.

C3 interaction has slightly more negative LUMO energy and smaller gap energy than $\mathrm{C} 2$, whereas $\mathrm{C} 2$ is more energetically stable than $\mathrm{C} 3$. Overall, both structures are effective, suitable for this combination and increase the activity of FPV. Further, FPV-HCQ interacted system fulfills all requirements of Lipinski, Veber and Egan rules, and it is predicted to be orally bioavailable.

These preliminary results may provide the lead for the design of more potent and selective COVID-19 drugs or vaccines. It is also worth noting that the possible combination of FPV and HCQ has the capacity to increase the effectiveness based on electron acceptance properties. Therefore, the combination might open new considerations and strategies against the COVID-19 and possible challenges in the future.

Supplementary Information The online version contains supplementary material available at https://doi.org/10.1007/s11696-021-01946-8.

Acknowledgements The authors acknowledge FenCluster system of Ege University for the calculations.

\section{Declarations}

Conflict of interests On behalf of all authors, the corresponding author states that there is no conflict of interest.

\section{References}

Alver Ö, Parlak C, Umar Y, Ramasami P (2019) DFT/QTAIM analysis of favipiravir adsorption on pristine and silicon doped $\mathrm{C} 20$ fullerenes. Main Group Met Chem 42:143-149

Astani EK, Heshmati E, Chen CJ, Hadipour NL (2016) A theoretical study on the characteristics of the intermolecular interactions in the active site of human androsteronesulphotransferase: DFT calculations of NQR and NMR parameters and QTAIM analysis. J Mol Grap Model 68:14-22

Ayala PY, Scuseria GE (1999) Linear scaling second-order MollerPlesset theory in the atomic orbital basis for large molecular systems. J Chem Phys 110:3660

Bader RFW (1990) Atoms in molecules-a quantum theory. Oxford University Press, Oxford

Boys SF, Bernardi F (1970) The calculation of small molecular interactions by the differences of separate total energies. some procedures with reduced errors. Mol. Phys. 19:553-566

Chang Y, Brewer NT, Rinas AC, Schmitt K, Smith JS (2009) Evaluating the impact of human papillomavirus vaccines. Vaccine 27:4355-4362

Colson P, Rolain JM, Raoult D (2020) Chloroquine for the 2019 novel coronavirus SARS-CoV-2. Int. J. Antimicrob. Agents. 15:105923

Coronavirus Worldometer, Covid-19 Coronavirus Pandemic, Last updated: May 19, 2021, https://www.worldometers.info/coron avirus/

Daina A, Michielin O, Zoete V (2017) SwissADME: a free web tool to evaluate pharmacokinetics, druglikeness and medicinal chemistry friendliness of small molecules. Sci Rep 7:42717

Dennington R, Keith T, Millam J (2009) Gauss View, Version 5. Semichem Inc., Shawnee Mission, KS

Fiore AE, Bridges CB, Cox NJ (2009) "Seasonal influenza vaccines" vaccines for pandemic influenza. Curr. Top. Microbiol. Immunol. Current Topics. Microbiol. Immunol. 333:43-82

Flynn E, Drug-Drug Interactions. 2007. xPharm: The Comprehensive Pharmacology Reference, Editör: S.J. Enna, D.B. Bylund, Boston: Elsevier. 1-3

Frisch MJ, Trucks GW, Schlegel HB et al. Gaussian 09, Revision A.1, Gaussian Inc, Wallingford, CT, 2009

Fukui K (1982) Role of frontier orbitals in chemical reactions. Science 218:747-754

Furuta Y, Takahashi K, Shiraki K, Sakamoto K, Smee DF, Barnard DL, Gowen BB, Julander JG, Morrey JD (2009) T-705 (favipiravir) and related compounds: novel broad-spectrum inhibitorsof RNA viral infections. Antiviral Res 82:95-102

Gautret P, Lagier JC, Parola P et al (2020) Hydroxychloroquine and azithromycin as a treatment of COVID-19: results of an openlabel non-randomized clinical trial. Int J Antimicrob Agents. https://doi.org/10.1016/j.ijantimicag

Hao J, Wang Z, Jin Q (2019) DFT study of structural, elastic, electronic and dielectric properties of blue phosphorus nanotubes. Sci Rep 9:11264

Jabłoński M (2019) On the uselessness of bond paths linking distant atoms and on the violation of the concept of privileged exchange channels. Chemistry Open 8:497-507

Kawakami J, Kakinami H, Matsushima N, Nakane A, Kitahara H, Nagaki M, Ito S (2013) Structure-activity relationship analysis for Antimicrobial activities of Tryptanthrin derivatives using quantum chemical calculations. J Comput Chem Jpn 12:109-112

Khorram R, Raissi H, Morsali A (2017) Assessment of solvent effects on the interaction of Carmustine drug with the pristine and $\mathrm{COOH}$-functionalized single-walled carbon nanotubes: A DFT perspective. J Mol Liq 240:87-97

Kumar S, Saini V, Maurya IK, Sindhu J, Kumari M, Kataria R, Kumar V (2018) Design, synthesis DFT, docking studies and ADME prediction of some new Coumarinyl linked Pyrazolylthiazoles: potential standalone or adjuvant Antimicrobial agents. PLOS One 13:1-23

Lai CC, Shih TP, Ko WC, Tang HJ, Hsueh PR (2020) Severe acute respiratory syndrome coronavirus 2 (SARS-CoV-2) and coronavirus disease-2019 (COVID-19): The epidemic and the challenges. Int. J. Antimicrob Agents. 17:105924 
Le TT, Andreadakis Z, Kumar A, Román RG, Tollefsen S, Saville M, Mayhew S (2020) The COVID-19 vaccine development landscape. Nat Rev Drug Discov 19:305-306

Liesegang TJ (2009) Varicella zoster virus vaccines: effective, but concerns linger. Can J Ophthalmol 44:379-384

Lu T, Chen F (2012) Multiwfn: A multifunctional wavefunction analyser. J Comput Chem 33:580-592

McKibbin W, Fernando R. The Global Macroeconomic Impacts of COVID-19: Seven Scenarios, CAMA working paper series 19 (2020)

Parlak C, Alver Ö (2017) A density functional theory investigation on amantadine drug interaction with pristine and $\mathrm{B}, \mathrm{Al}, \mathrm{Si}, \mathrm{Ga}, \mathrm{Ge}$ doped C60 fullerenes. Chem Phys Lett 678:85-90

Parr RG, Szentpály LV, Liu S (1999) Electrophilicity Index. J Am Chem Soc 121:1922-1924

Pierens GK, Venkatachalam TK, Reutens DC (2017) NMR and DFT investigations of structure of colchicine in various solvents including density functional theory calculations. Sci Rep 7:5605

Reed AE, Curtiss LA, Weinhold F (1988) Intermolecular interactions from a natural bond orbital, donor-acceptor viewpoint. Chem Rev 88:899-926

Rhyman L, Tursun M, Abdallah HH, Choong YS, Parlak C, Kharkar P, Ramasami P (2018) Theoretical investigation of the derivatives of favipiravir (T-705) as potential drugs for Ebola virus. Phys. Sci. Rev. 3:20170198

Rozas I, Alkorta I, Elguero J (2000) Behavior of ylides containing N, $\mathrm{O}$, and $\mathrm{C}$ atoms as hydrogen bond acceptors. J Am Chem Soc 122:11154-11161

Samsonowicz M, Regulska E, Kowczyk-Sadowy M, Butarewicz A, Lewandowski W (2017) The study on molecular structure and microbiological activity of alkali metal 3-hydroxyphenylycetates. J Mol Struct 1146:755-765

Samsonowicz M, Regulska E, Świsłocka R, Butarewicz A (2018) Molecular structure and microbiological activity of alkali metal 3,4-dihydroxyphenylacetates. J Saudi Chem Soc 22:896-907

Savin A, Nesper R, Wengert S, Fassler TF (1997) ELF: The electron localization function. Angew Chem Int Ed 36:1809-1832

Shahbazian S (2018) Why bond critical points are not "bond" critical points. Chem Eur J 24:5401-5405
Simon S, Duran M, Dannenberg J (1996) How does basis set superposition error change the potential surfaces for hydrogen-bonded dimers? J Chem Phys 105:11024-11031

Singh AK, Singh A, Shaikh A, Singh R, Misra A (2020a) Chloroquine and hydroxychloroquine in the treatment of COVID-19with or without diabetes: a systematic search and a narrative reviewwith a special reference to India and other developing countries, Diabetes Metabolic Syndrome: Clin. Res Rev 14:241-246

Singh A, Shaikh A, Singh R, Singh AK (2020b) COVID-19: From bench to bed side, diabetes metabolic syndrome: Clin Res Rev $14: 277-281$

Swiss ADME. 2017. A free web tool to evaluate pharmacokinetics, drug-likeness and medicinal chemistry friendliness of small molecules. Sci. Rep. 742717

Tomasi J, Mennucci B, Cammi R (2005) Quantum mechanical continuum solvation models. Chem Rev 105:2999-3093

Wong JEL, Leo YS, Tan CC (2020) COVID-19 in Singapore-current experience critical global issues that require attention and action. JAMA 323:1243-1244

Wu A, Peng Y, Huang B, Ding X, Wang X, Niu P, Meng J, Zhu Z, Zhang Z, Wang J (2020) Genome composition and divergence of the novel coronavirus (2019-nCoV) originating in China. Cell Host Microbe 27:325-328

Zhai F, Wei H, Liu Y, Hu F (2019) Theoretical explanation for the pharmaceutical incompatibility through the cooperativity effect of the drug-drug intermolecular interactions in the phenobarbital paracetamol H2O complex. J Mol Model 25:181

Zhang SX, Wang Y, Rauch A, Wei F. Health, Distress and Life Satisfaction of People in China One Month into the COVID-19 Outbreak (3/12/2020). Available at SSRN: https://ssrn.com/abstract= 3555216 or https://doi.org/10.2139/ssrn.3555216

Zheng YZ, Zhou Y, Liang Q, Chen DF, Guo R, Lai RC (2016) Hydrogen-bonding Interactions between Apigenin and Ethanol/water: a theoretical study. Sci Rep 6:34647

Publisher's Note Springer Nature remains neutral with regard to jurisdictional claims in published maps and institutional affiliations.

\section{Authors and Affiliations}

\section{Cemal Parlak $^{1}$ - Özgür Alver ${ }^{2} \cdot$ Cecil Naphtaly Moro Ouma ${ }^{3} \cdot$ Lydia Rhyman $^{4,5} \cdot$ Ponnadurai Ramasami $^{4,5}$}

1 Department of Physics, Science Faculty, Ege University, Izmir 35100, Turkey

2 Department of Physics, Science Faculty, Eskisehir Technical University, Eskisehir, Turkey

3 HySA-Infrastructure CoC, Faculty of Engineering, North-West University, Private Bag X6001, Potchefstroom 2531, South Africa
4 Computational Chemistry Group, Department of Chemistry, Faculty of Science, University of Mauritius, Réduit 80837, Mauritius

5 Centre for Natural Product Research, Department of Chemical Sciences, University of Johannesburg, Doornfontein Campus, Johannesburg 2028, South Africa 